\title{
THE PRESENT POSITION OF CHEMOTHERAPY IN TUBERCULOSIS
}

\author{
By JoHN C. Roberts, M.D., M.R.C.P. \\ Consultant Physician, Harefield Hospital, Harefield, Middlesex
}

Considerable advances have been made in recent years in the use of chemotherapy in tuberculosis. IVhile alterations in technique are constantly under review the following summarises the present position.

Combinations of drugs and treatment schedules that combine maximum benefit with minimal drug toxicity and bacterial resistance, have been the object in much research all over the world.

The drugs commonly in use are streptomycin, para-amino-salicylic acid (PAS), isonicotinic acid hydrazide (INAH) and the thiosemicarbazones.

It is now generally agreed that combinations of drugs are more effective than one or other antibiotic alone, and also such combinations delay the resistance of the tubercle bacillus to the individual drugs.

The toxic effects of the drugs used will first be considered.

\section{Streptomycin}

Toxic effects are less common now that the dosage is reduced to $\mathrm{I} g$. per day rather than the original dosage of 2 to $3 \mathrm{~g}$. per day. A survey by the Tuberculosis Association (1952) confirms this. Dihydrostreptomycin may cause deafness whereas streptomycin sulphate appears to have an effect on the vestibular function, Bignall et al. discuss these effects. Both these complications are now less common with lower dosage and improved purity of the drugs. Dihydrostreptomycin should, however, be avoided intrathecally owing to the increased risk of deafnesss. The labyrinthine disturbance tends to recover in time, but deafness when it occurs is usually permanent. Heck et al. suggest that a mixture of equal quantities of dihydrostreptomycin and streptomycin sulphate reduce the risk of neurotoxic effect. These disturbances are of importance and call for care in returning a patient to an occupation where the disability may be dangerous. Other toxic effects are skin reactions-urticarial and erythematous, lym- phadenopathy, fever, nausea and vomiting. Sumner (1949) drew attention to the development of a vitamin $B$ deficiency and recommended prophylactic administration of vitamin B to patients having streptomycin. Local contact of the drug may sensitize the skin and has been recorded in nurses through giving injections without wearing gloves or taking precautions to avoid skin contact. Desensitization is usually possible and is carried out by starting with small injections, such as $10 \mathrm{mg}$., and slowly working up to I g. Crofton (1952) gives a good account of this and in one case showed that desensitization to dihydrostreptomycin does not desensitize against the sulphate. He took some 35 days in one case and 23 weeks over the other to desensitize. He also used antihistamine preparations. The writer can confirm these results.

\section{Para-amino-salicylic Acid}

The toxic effects of PAS are well summarized in a Lancet annotation (1953). Nausea, vomiting and diarrhoea are the commonest. Pyrexia, skin rashes, liver damage with associated defects and jaundice occur. Myxoedema, anuria, mental changes, glandular enlargement and splenomegaly are also recorded. The allergy may be more general and effect sensitization to sulphonamides and streptomycin as well. Profound psychic disturbances have been described by Pugh et al. (1952), who consider that they are due to salicylate stimulation of the pituitary gland, resulting after a time in pituitary exhaustion and deficiency of cortin. In giving PAS it is wise to give it after a meal in liquid torm or, in some cases, enteric coated granules or cachets washed down with a drink of milk are preferred by the patient. Many preparations are available which attempt to prevent or minimize the nausea. Adreno-corticotrophin (ACTH) has been successfully used to overcome the allergy to PAS (Houghton, I952).

As there is some evidence that AC'TH and cortisone when used alone have an adverse effect on 
tubercle, and also as these substances themselves have their own unpleasant side effects, great care is necessary in their use. It is important when using ACTH or cortisone to make sure that the bacillus is sensitive to the antibiotic employed before either is used. It is probably safer where there is no urgency to desensitize, as described by Crofton.

\section{Isonicotinic Acid Hydrazide}

In the dosage used, namely 200 to $400 \mathrm{mg}$. daily, this seems to have little toxicity and is very convenient and easy to take. Damage to the liver and jaundice with haemopoetic depression has been described in animal experiments (Rubin et al., 1952). Marked euphoria and convulsions are also recorded. Some state that a diminished sugar tolerance occurs which is of importance in diabetics (Luntz and Smith, I953). The writer has not experienced difficulty in using this drug in diabetics.

\section{Thiosemicarbazones}

These are not used very frequently now. The most effective and least toxic is said to be ethizone (TB.3). They have, however, toxic effects and may cause liver damage and disturbance of the haemopoetic tissues. An account of the use of this drug in genito-urinary tuberculosis is given by Ross-Cosbie et al. (I 953) who gave up to $200 \mathrm{mg}$. daily for six months continuously, combined with alternating courses of PAS and dihydrostreptomycin.

\section{Resistance}

The resistance of tubercle bacilli to the various antibiotics used has been the subject of much discussion. It is customary to assess the sensitivity by laboratory tests at the onset of treatment without necessarily waiting for the result, which takes some weeks, before starting treatment. A few resistant strains may be found in the sputum of patients who have had no previous chemotherapy. The organisms in the sputum may, during treatment, show increasing resistance in laboratory examinations. Although laboratory investigations have shown resistant strains in a particular case, the patient may still continue to respond to the drugs. It must be appreciated that the specimen examined is only a sample, and it cannot be presumed that all the bacilli in the various lesions are equally sensitive or resistant. Resistance has, however, clinical significance in that some patients with resistant strains fail to respond to further chemotherapy. Cavitated lesions are more liable to develop resistant strains than non-cavitated lesions.

In the early trials using streptomycin in dosage of $2 \mathrm{~g}$. daily, resistant strains began to appear at about the end of the second month of treatment, and after four months resistant strains were found in 85 per cent. of those whose sputum remained positive. Attempts to reduce this incidence by reducing the dose and trying intermittent courses of treatment met with some success. The addition of PAS or INAH not only proved more effective in treatment but also in preventing or delaying the emergence of resistance to each of the drugs used. PAS used alone has a beneficial effect but not to the same extent as streptomycin. Resistance develops but usually takes longer to do so than with streptomycin. Turnbull et al. (1953) have shown when resistance to PAS has occurred it is useless to use it with streptomycin in the hope of delaying the development of streptomycin resistance.

INAH has many advantages over PAS but resistant strains develop quickly. After six weeks' treatment on a dosage of $200 \mathrm{mg}$. daily in those patients whose sputum still remains positive, 60 per cent. show bacilli resistant to INAH. It has been shown experimentally by Barnett et al. (1953) that INAH resistant strains produced in vitro and in mice reverted to sensitivity when contact with the drug was stopped after one subculture. They also showed that resistant strains from human subjects tended to regain their sensitivity during three sub-cultures in the absence of the drug. Mice and guinea pigs inoculated with non-resistant strains were completely protected. from developing tuberculosis by a dose of $20 \mathrm{mg}$ of INAH per kg. Even when the mice and guinea pigs were inoculated with resistant strains a similar dose of the drug increased the survival of the animals considerably. Joiner et al. (1952) showed that strains resistant to INAH from patients tended to revert to sensitivity after treatment was stopped. This latter observation has not been confirmed in the recent Medical Research Council trials, where no alteration in resistance was found after three months following cessation of treatment. Treatment with INAH alone proved disappointing in that initial improvement was not maintained. The combination of streptomycin and INAH has been shown to have as good if not better therapeutic effect as streptomycin and PAS, and the resistance of organisms to both drugs is delayed. The usual dose of INAH for an adult is 200 to $400 \mathrm{mg}$. a day. Using INAH and streptomycin in a series of cases it was found that of those with $N$ positive sputum cultures at the end of three months' treatment, only i I per cent. showed resistant strains, whereas using INAH alone the o figure rose to 64 per cent. Detailed investigations on these effects are continuing in the Medical ? Research Council trial (Lancet, 1953).

Before discussing the chemotherapeutic usage in individual fields, certain general principles 
should be observed. A careful assessment of the case is essential, as chemotherapy is only one factor in the treatment, and its use should fit in with the general plan. Full bacterial examination with sensitivity tests should be carried out, the material being obtained before treatment with the drugs is initiated, although in most cases it is not practicable to await the results before commencing treatment. In the present state of our knowledge, a single antibiotic should never be used alone. Streptomycin should always be combined with either PAS or INAH or both. There is some evidence that PAS and INAH will prove an efficient combination, but there is as yet insufficient information on this point. There is also some evidence that streptomycin used on alternate days in $2 \mathrm{~g}$. dosage together with INAH, $150 \mathrm{mg}$. daily, has produced good results and also inhibited the development of the resistant strains.

\section{Clinical Applications}

\section{Pulmonary Tuberculosis}

(a) Primary tuberculosis.- Some authorities advise chemotherapy as a routine in children under the age of two in view of the greater seriousness of the condition at this age. Lorber (1950) has shown that streptomycin has little effect on simple primary lesions. The writer agrees with this view and reserves antibiotic treatment for complicated lesions, such as progressive primary lesions with cavitation. The drugs also should be used to cover surgical procedures such as tonsillectomy or bronchoscopic examinations or resections in patients with primary tuberculosis. The dosage of streptomycin in infants depends on the weight-a usual guide is $.02 \mathrm{~g}$. of streptomycin per $\mathrm{lb}$. body weight. The drug should always be combined with PAS or INAH. The latter is more easily tolerated by infants and children. Suggested dosage is $100 \mathrm{mg}$. daily in infants under two, $150 \mathrm{mg}$. between the ages of two and ten and $200 \mathrm{mg}$. at the age of ten or over.

(b) Miliary tuberculosis. It is necessary here for treatment to be prolonged. In adults a gramme of streptomycin a day is now considered adequate. This is combined with PAS, $20 \mathrm{~g}$. a day in divided dosage, and INAH, 200 to $400 \mathrm{mg}$. daily. Where the PAS is not well tolerated the dose may have to be reduced or the drug omitted altogether and INAH used alone with the streptomycin. Even though clearing of the lesions occurs in three months it is wise to continue treatment for at least six months and preferably nine months to a year. The streptomycin can be given less frequently atter three months if progress is good. PAS and INAH may be used alternatively with the streptomycin. Some prefer to use the three drugs together. There is no rigid rule but it is wise to insist on prolonged treatment to prevent relapse. Routine lumbar punctures should be performed and if the fluid becomes abnormal the case should be treated as one of tuberculous meningitis.

(c) Bronchogenic tuberculosis. The best results are undoubtedly obtained in recent active disease. Chronic disease is often benefited but relapse is common. Resistance of the organism to the antibiotic and failure of the patient to respond is more likely to develop in those with chronic thick-walled cavitated disease.

It cannot be too strongly stressed that general measures are very important in all forms of tuberculous disease.

In early active disease a frequent course consists of three months of streptomycin combined with INAH or PAS or a combination of the three. Some use daily streptomycin for the first six weeks and then alternate days for the next six weeks. A single injection daily of a gramme is considered adequate. The oral antibiotics are given daily; $20 \mathrm{~g}$. of PAS is usually given in 24 hours in $5-\mathrm{g}$. doses, if this is not well tolerated the dose may be reduced to 16 or even to $10 \mathrm{~g}$.; 200 to $400 \mathrm{mg}$. of INAH is given daily, divided into two or three doses. After three months some give no further drugs if improvement has occurred, but resume chemotherapy if the improvement is not maintained or there is any evidence of a relapse. In America there is a greater tendency to use continuous chemotherapy for tuberculosis, even when surgical treatment is envisaged. One year or more continuous treatment with antibiotics is not uncommon.

In chronic ambulant cases courses of antibiotics may improve the patient clinically and also sterilize the sputum for some time. The risk of dissemination of resistant strains does not appear to have been as great as was expected and it does not seem justifiable to withhold treatment in these cases for this reason. Again some use intermittent courses of three months, some reserve treatment for the times when the disease shows signs of activity and others favour more prolonged treatment. Little is known of the end results in this group.

In the surgical treatment of pulmonary tuberculosis, particularly in those patients subjected to resection, all agree on the desirability of covering the procedure with antibiotics. Most patients have had some months or years of conservative treatment first and the majority have had one or more courses of antibiotic treatment. The operative cover is usually started one or two weeks prior to surgery and continued for four to six weeks afterwards, daily streptomycin (I g.) with PAS or INAH by mouth being given in addition. Some then reduce the frequency of the streptomycin and 
continue the antibiotic treatment for a further six weeks or so. Much depends on the individual case and the nature of the surgery. The sensitivity of the bacilli to antibiotics in influencing a surgical decision is a matter of opinion. Some surgeons hesitate to undertake pulmonary resection unless the bacillus is known to be sensitive to at least one of the antibiotics.

Tuberculosis of the larynx and of the bronchi show good response to chemotherapy. There is a tendency for fibrosis to occur with streptomycin, and bronchial stricture may develop. This complication is of much importance when it occurs in a main bronchus or one of the major divisions, since infected secretions may be held up beyond the stricture and produce progressive lung destruction. It is therefore of importance to remember this possibility in treating tracheobronchial tuberculosis with streptomycin.

Laryngeal tuberculosis if treated early shows a dramatic response. Symptoms may disappear in 24 hours and the writer has observed complete resolution occur in as short a period as a week.

(d) Pleural effusion. There is no convincing evidence that antibiotics influence the course of pleural effusion or prevent sequelae. But some physicians give a course during the acute phase. In tuberculous empyema good results have been reported using streptomycin and PAS both locally and systemically. In early empyemas the response is very good.

\section{Tuberculous Meningitis}

It is in this disease that the most spectacular advances have been achieved. The general tendency has been to prolong treatment. McCarthy and Mann (1950) recommend three months intramuscular streptomycin combined with three months intrathecal streptomycin. The intramuscular dose depends on the weight of the child; the intrathecal dose varies between .05 and .I $\mathrm{g}$. On this regime recoveries up to about 50 per cent. have been reported. It is usual to combine PAS daily with streptomycin or, more recently, INAH. The latter is much easier to administer.

Anderson et al. (1953) describe a few cases in which INAH was the main treatment. Intramuscular and intrathecal streptomycin was given for one week only in a few cases, and for one month in two cases. INAH was continued orally for 16 weeks. One case which had relapsed from previous treatment was treated entirely by INAH orally for 16 weeks. All cases were said to show remarkable response. Ritchie et al. (1953) suggested that INAH is more diffusible than streptomycin, having a more rapid action on the bacilli and promoting increased vascularity and resolution of the lesions in miliary disease and meningitis.
Juan Torres-Gost (1953) reports on 100 cases with six deaths only. He uses intramuscular strepto- ํㅡㄱ mycin in dosage of $.3 \mathrm{~g}$. daily in infants under two, increasing the dose to $.5 \mathrm{~g}$. in severe cases. Between the ages of two and ten I g. daily in two doses for ten days followed by $.5 \mathrm{~g}$. daily until the fever subsides. In adults I g. daily is given in two doses until the pyrexia subsides, combining this treatment with oral and intrathecal INAH. In क children under two he gives $150 \mathrm{mg}$. of INAH daily, the dose increasing with age until the adult dose of $400 \mathrm{mg}$. is reached. He also gives $10 \mathrm{mg}$. of intrathecal INAH daily to infants under two, $\stackrel{\circ}{\circ}$ increasing the dose to a maximum of $50 \mathrm{mg} \cdot \overrightarrow{\vec{H}}$ intrathecally daily for adults. A total dosage of 30 to $45 \mathrm{~g}$. of streptomycin over three months, 25 to $30 \mathrm{~g}$. of INAH orally and 55 intrathecal in -3.3 jections is the suggested course. In the first month the intrathecal injections are daily, in the second $\stackrel{\odot}{\omega}$ month on alternate days and in the third month + every third day. Torres-Gost suggests that if the cell count in the cerebrospinal fluid is higher thanc 20 after four months' completion of treatment, a응 second course of streptomycin combined with oraland intravenous INAH should be given.

Fletcher (I953) has shown that adequate levels in the spinal fluid of INAH are obtained in $\rightarrow$ tuberculous meningitis on an oral dosage of 2000 mg. per day. It is therefore difficult to see intrathecal INAH should be necessary. Int thecal streptomycin enhances the prospect of deat-s ness particularly if dihydrostreptomycin sulphateo is used, and for this reason alone any satisfactoryo treatment which avoids the use of intrathecal $\mathbb{Q}$ streptomycin is desirable.

Frequent lumbar punctures even when carried 3 out expertly in a sedated child or adult, tend to impose considerable strain on the patient after $\bar{p}$. time and it is to be hoped that the early results with INAH orally combined with streptomycin intra muscularly will prove an adequate treatment in' this condition. It would seem reasonable to com mence on these lines and only to add intrathecab streptomycin if progress does not appear satis factory.

One of the problems in tuberculous meningitis is the occurrence of spinal block. Cathie (1950르. used intrathecal streptokinase in an attempt to liquify the exudate at the base of the brain to prevent the development of obstruction. Un fortunately reactions to this treatment occur and the writer had to abandon its use in one case of account of severe hypersensitivity changes.

Tuberculin intrathecally has been usec్ by Smith et al. (1950). The initial dose is $.0000035 \mathrm{mg}$., which is increased every day. The dosage and rate of increase will depend on the re $\frac{O}{\mathbb{D}}$ action produced. These reactions consist of ar? 
intensification of the meningeal signs which are sometimes very severe, with hyperpyrexia and coma due to a great rise in intracranial pressure. The treatment is reserved for cases not improving on routine lines and should only be carried out in special units. Some of their results were spectacular.

Some authorities make burr holes as a routine measure so that if spinal block occurs and the cerebralspinal fluid pressure becomes high this can be relieved by tapping the lateral ventricles by catheters and introducing antibiotics through this route. Most authorities regard a spinal fluid that is not normal or showing progessive return to normal after one of the courses of treatment outlined above as an indication for further active treatment. Luminal should be given to all cases of meningitis for a period of two years to minimize the chance of epileptic fits occurring. Relapse is not likely if the spinal fluid has become completely normal.

\section{Bone and Foint Tuberculosis}

It is the experience of most workers that the cases associated with toxaemia and sinuses respond best. The closed spinal lesion without fever or toxaemia shows little response. Our American colleagues, Evans et al. (1952), favour long courses of antibiotic treatment with active surgical intervention under streptomycin cover. Evans considers that streptomycin should only be used for critical episodes in the patient's illness. They all report improvement in tubercular synovitis. In England chemotherapy is most commonly used in bone and joint tuberculosis where there is toxaemia with progressive destruction of bones or joints, where there are sinuses, or to cover surgical intervention. The drug should always be combined with INAH or PAS. There is no uniform agreement as to the length of the treatment.

\section{Genito-Urinary Tuberculosis}

Ross et al. report on the treatment of renal tuberculosis by using a combination of ethizone, $200 \mathrm{mg}$. daily for six months, combined with PAS alternating with dihydrostreptomycin. The PAS is given daily for 25 days and is followed by the dihydrostreptomycin for 30 days. They also make the urine alkaline with a citrate mixture. The treatment is combined with surgical removal where indicated. Dick (1953) reports on the good effect of INAH and on histological evidence states the drug increases vascularity in the lesions favouring absorption. One of the disadvantages of streptomycin therapy in tuberculous cystitis is that fibrosis of the bladder occurs which is associated with a reduction in size and sometimes some degree of ureteric obstruction by fibrosis in the region of the ureteric orifices. Most surgical authorities use chemotherapy as an adjuvant to surgery. Chemotherapy used as the sole method of treatment has proved disappointing. Some attempts are being made, however, to perform segmental renal resections under antibiotic cover to preserve as much renal tissue as possible.

Genital Tuberculosis in the Female. This is being diagnosed more frequently since investigations in sterility clinics show an appreciable number of women complaining of sterility have tuberculous infection of the tubes, ovaries, and sometimes the uterus. Surgical treatment is of necessity rather radical and there is a tendency to give antibiotic treatment a more lengthy trial. Burns et al. (1953), treating 43 cases with INAH followed by streptomycin and PAS for a total period of four months, report that 40 out of 43 cases showed no evidence of infection at the end of treatment. One patient became pregnant.

\section{Pericardial Effusion}

This has been treated with systemic streptomycin and PAS and in some cases intrapericardial streptomycin has been used. Pericardial resection has been successfully carried out under streptomycin cover in quiescent cases.

\section{Lupus Vulgaris}

This is said to be influenced favourably by INAH.

With increasing research there is little doubt that more and more anti-tuberculous preparations will be evolved and further light will be thrown on the most effective combinations. It must not be forgotten that at all times there will be of necessity individual variations both in the reaction of the patient and in the views of the physicians and surgeons on treatment. It is of the greatest importance that indiscriminate use of anti-tuberculous drugs should be avoided. Treatment should only be carried out after careful assessment of the problem as a whole; wherever possible complete bacteriological examination should be carried out before treatment starts and sensitivity tests arranged. It is obviously not practicable to wait for the results before commencing treatment. There are still many problems with regard to the effect of the drugs and particularly with regard to the development of, and the significance of, resistant strains which have yet to be cleared up. It may be said that in general there is a tendency to use more prolonged antibiotic treatment and that in the surgical treatment of tuberculosis the necessity for careful pre- and post-operative sanatorium regimes for longer periods is being accepted.

Continued on page 293. 


\section{Streptomycin, para-amino salicylic acid and isoniazide}

In the acute forms of tuberculosis antibiotics are of great value. It is doubtful whether they have any definite use in the treatment of large caseating glands or chronic caseating pneumonia. Neither the size of the caseating lesions nor the rate of healing is dramatically influenced. There is not at present sufficient evidence to assess their value in those cases where ulceration of a bronchus is present.

Since it is probable that haematogenous infection to a greater or less degree arises in many primary lesions, especially in infants, antibiotics are given by some physicians to young children who have evidence of recent infection with definite primary lesions, hoping in this way to reduce the possibility of miliary lesions both evident and occult.

\section{Bronchoscopy and Surgical Treatment}

Treatment when a bronchus has become involved in the primary lesion presents many problems. It may be necessary, though rarely, as an emergency measure to clear the lumen of a bronchus of tuberculous material when stridor is marked and endangering a child's life. Bronchoscopic removal of the material may be attempted or, alternatively, thoracotomy and removal of the mass of glands invading the bronchial wall. Bronchoscopy may be indicated for removal by suction of pus from bronchi, beyond the partial obstruction, which have become secondarily infected. Richards and Blair ${ }^{6}$ recommend routine bronchoscopy in order to reduce the possibility of bronchiectasis developing in the collapsed lobe.

How to treat the collapsed lobe is still a debatable point. That bronchiectasis may result from such collapse is an established fact. As yet the number of cases with collapse which develop bronchiectasis with symptoms in later life is not known. Prevention and treatment of secondary infection in the collapsed segment is no doubt of importance. The problem of the collapsed lobe is at present being dealt with usually in one of three ways. Expectant treatment is advocated by some on the assumption that the majority heal sufficiently to allow complete or partial re-expansion of the collapsed lobe and, in only a few cases, will bronchiectasis develop later; others recommend resection of the collapsed lobe, or segment, either at an early stage or after a period of a year or more if there has not been complete recovery, so preventing future trouble in an unexpanded or partially re-expanded segment. The third form of treatment is thoracotomy and removal of the mass of caseating glands in order to prevent possible irreversible collapse and development of bronchiectasis.

The treatment of chronic caseating lesions occupying large areas of the lung is also varied. With our present knowledge of this type of lesion it is not possible to say how many of them heal completely, although apparently there are some which do so. It is clear that many of them remain in a chronic state for a year or more. It has been suggested that parts of these lesions may remain unhealed and, in later life, give rise to clinical pulmonary tuberculosis. On this assumption, it is suggested that removal of such caseating lung lesions is preferable to a 'wait and see' policy.? On the one hand there is the risk of the operation and the possible disadvantage to a child of the loss of a lobe or even a complete lung and, on the other, the disadvantage of a long period of treatment probably away from home and the possible relationship of these lesions to clinical tuberculosis in later life.

\section{REFERENCES}

1. MEDICAL RESEARCH COUNCIL, National Tuberculin Survey (1952), Lancet, i, 775 .

2. HYGE, T. V. (1945), Ugesk. f. lacger, 105, 779

3. CAMMOCK, R. M., and MILLER, F. J. W. (1953), Lancet, i, 158.

4. BEAVEN, P. W. (1950), Dis. of Chest, 17, 280.

5. THOMPSON, B. C. (1952), Proc. Roy. Soc. Med., 45, 741 .

7. THOMAS, D. (1952), Proc. Roy. Soc. Med., 45, 743.

Continuation of Bibliography-Fohn C. Roberts, M.D., M.R.C.P., from page 287.

\section{BIBLIOGRAPHY}

MAHER-LOUGHNAN, G. P. (1952), Tubercle, 33, 342.

BIGNALL, J. R., CROFTON, J. W., and THÖMAS, J. A. B. (1951), Brit. med. F, i, 554 . Admin. Conf. on Chemotherapy of Tuberculosis.

SUMNER, J. (1949), Tubercle, 3, 62.

CROFTON, J. (1952), Brit. med. $\mathscr{F}$., ii, 1014.

ANNOTATION (1953), Lancet, i, $23 \mathrm{I}$. JONES, E.' R. (1952), Tubercle, 33, 369 .

HOUGHTON, L. E. (1952), Ibid., 33, 381.

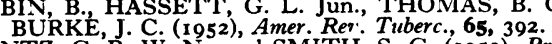

LUNTZ, G.' R. W. N., and SMITH, S. G. (1953), Brit. med. F.,

ROSS-COSBIE, J., GOW, J. G., and ST. HILL, C. A. (1953),

Ibid.ji, 90I.
TURNBULL, F. W. A., WALLACE, A. T., STEWART, SHEILA, and CROFTON, J. W. (I953), Ibid., i, I 244

MCCARTHY, D., and MANN, T. P. (1950), Ibid., i, 34 I.
BARNETT, MARGARET, BUSHBY, S. R. M., and MITCHESÓN, D. A. (I953), Lancet, i, 314.
JOINER, C. L., MACLEAN, K. S.,PRITCHARD, E. K., ANDER-
SON, K., COLLARD, P., KING, M. B., and KNOX, R. (rON2, Kbid., ii, 843.

MEDICAL RESEARCH COUNCIL REPORT No. 4 (1953), Ibid., ii, 217 .

LORBER, J. (1950), Ibid., i, 389.

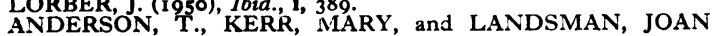
(I953), Ibid., ii, 691 .

RITCHIE, M., 'TAYLOR, R. M., and DICK, J. C. (1953), Ibid., ii, 419.

TORRES-GOST, J. (1953), Ibid., ii, 693

FLETCHER, A. P. (1953), Ibid., ii, 694 .

CA'THIE, L. A. P. (1950), Ibid., i, 441 .

SMITH, HONOR V., and VOLLƯM, R. L. (1950), Ibid., ii, 275.

EVANS, E. T. (1952), Ұ. Bone and Foint Surg., 2, 267.

DEROY, M. S., and FISHER, H. (1952), Ibid., 2, 299.

DICK, J. C. (1953), Lancet, i, 808.

BURNS, T., SMITH, H. G. M., and SNAITH, L. M. (1953), Ibid., i, 81 7 . 